\title{
Splitting strategies for video images processing in medical data grid
}

\begin{abstract}
There has a lot of improvements had been done on the diagnostic tools. Due to these improvements, most of the medical images are now migrating from 1D (e.g. cardiograms and encephalograms) and 2D (e.g. x-rays) images to extended 3D (e.g. tomography) and eventually 4D (3 spatial dimension + time) image. This migration process has let the volume of medical image become larger and become difficult to store. To overcome this kind of problems, most of the hospitals start to integrate the grid technology into their medical storage system. In this paper, besides presented the current related research projects in the medical field, we also briefly discussed on the details of medical data grid, and the strategies and techniques used in the video and image processing area. From the discussion, based on the parameters of the initial delay and the deadline miss, we found that the Fibonacci-based splitting strategy is the most appropriate strategy for used in the video images processing.
\end{abstract}

Keyword: Grid computing; Medical data management; Digital medical images; Splitting strategies; Image processing; Video processing 